\title{
Enhancing the Role of Government, Non-Profits, Universities, and Resident Associations as Valuable Community Resources to Advance Equity, Access, Diversity, and Inclusion
}

\author{
Ivis García ${ }^{1, *}$ and April Jackson ${ }^{2}$ (D) \\ 1 Department of City and Metropolitan Planning, University of Utah, Salt Lake City, UT 84112, USA \\ 2 Department of Urban and Regional Planning, Florida State University, Tallahassee, FL 32306, USA; \\ ajackson5@fsu.edu \\ * Correspondence: ivis.garcia@utah.edu
}

check for

updates

Citation: García, I.; Jackson, A.

Enhancing the Role of Government, Non-Profits, Universities, and Resident Associations as Valuable Community Resources to Advance Equity, Access, Diversity, and Inclusion. Societies 2021, 11, 33 https://doi.org/10.3390/soc11020033

Received: 6 April 2021

Accepted: 7 April 2021

Published: 10 April 2021

Publisher's Note: MDPI stays neutral with regard to jurisdictional claims in published maps and institutional affiliations.

Copyright: (c) 2021 by the authors. Licensee MDPI, Basel, Switzerland. This article is an open access article distributed under the terms and conditions of the Creative Commons Attribution (CC BY) license (https:// creativecommons.org/licenses/by/ $4.0 /)$

\section{About Asset-Based Community Development (ABCD)}

Asset-based community development $(A B C D)$ recognizes the value of six local assets: (1) individuals or community residents, (2) neighborhood resident associations (3) local institutions (e.g., government, non-profits, and universities) (4), physical space (e.g., parks, vacant lots, etc.), (5) economy and exchange (e.g., business development, barter, etc.), and (6) culture, history, and stories. ABCD draws upon these assets to build stronger and more sustainable communities [1] (see Figure 1).

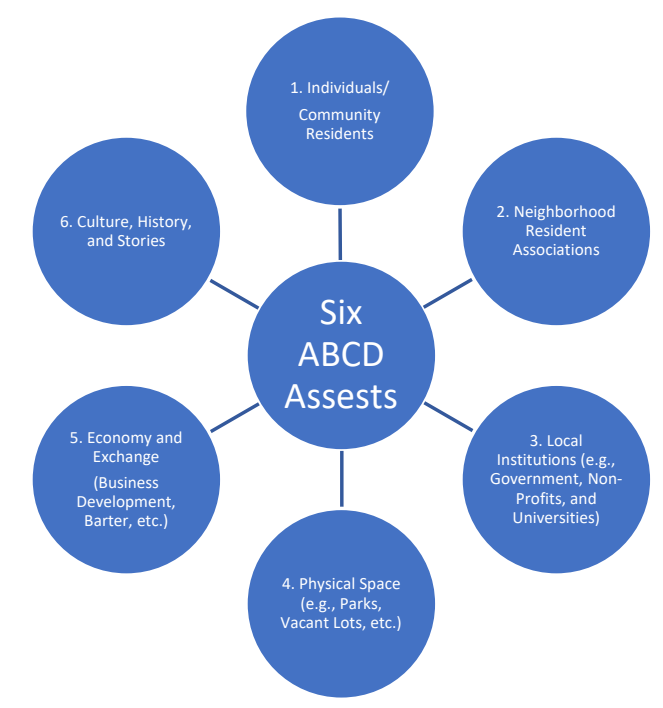

Figure 1. The six assets of asset-based community development (ABCD). Source: Authors.

In their book "Building Communities from the Inside Out: A Path Toward Finding and Mobilizing a Community's Assets" (1993), John McKnight and Jody Kretzmann started a movement on how to achieve long-lasting neighborhood change based on mapping and mobilizing community resources or assets. In this book, they outline three basic principles of what they coined as "asset-based community development" or ABCD for short. ABCD is the foundation of building power within communities, and it asks the following questions to mobilize assets: (1) What assets can I use to make this community better? (the gifts of the individuals or residents); (2) What assets do my neighbors have that can make our community better? (the gifts of groups of individuals aka resident associations); (3) What assets do the government, non-profits, universities, and other organizations in my local area have that can make my community better? (the gift of institutions). These three 
questions in Figure 2 can be used to build on the gifts (assets) of residents, neighborhood associations, and local institutions.
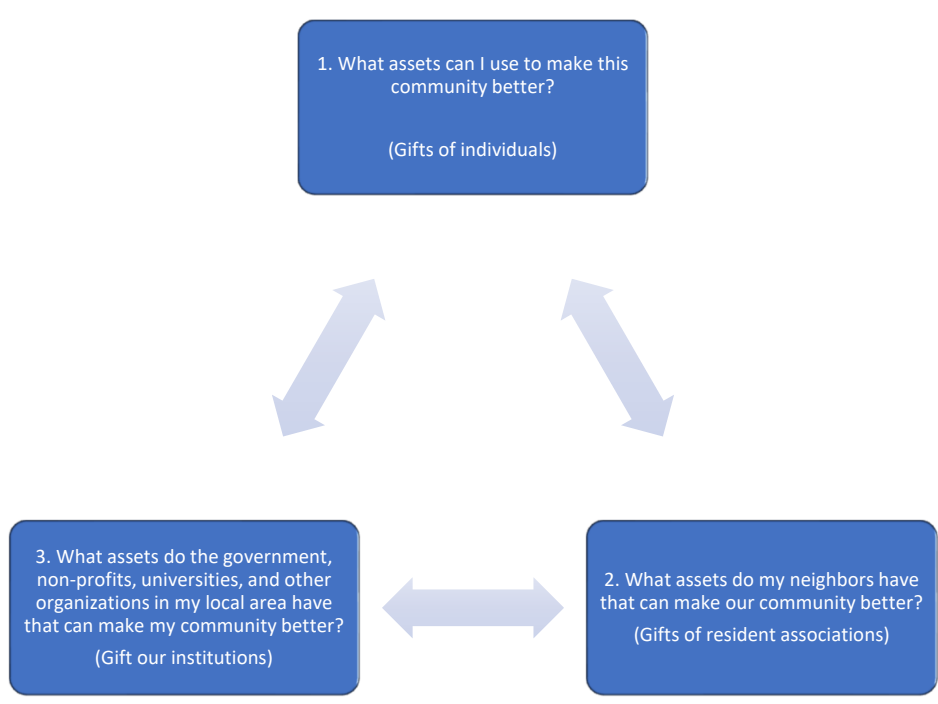

Figure 2. Three basic questions to mobilize community assets. Source: Authors.

McKnight and Kretzmann describe three basic principles for an alternative community plan that is not based on the needs or deficits of those who are low-income, older adults, young people, etc. Instead, ABCD seeks to empower these individuals by changing the model from being top-down to being bottom-up by employing three basic principles of community development, namely: (1) asset-based, that is, starting with what the neighborhood already has, such as the capacities, skills, or resources of residents, associations, institutions, as well as their current local economy, physical space, and community stories of success, rather than what the neighborhood needs and what it is lacking that needs to be fixed; (2) internally focused, meaning that local residents and associations with the help of institutions are the ones that should drive the change in neighborhoods; (3) relationship driven, which means seeking to catalyze change through mutually beneficial partnerships between diverse community partners (Figure 3).

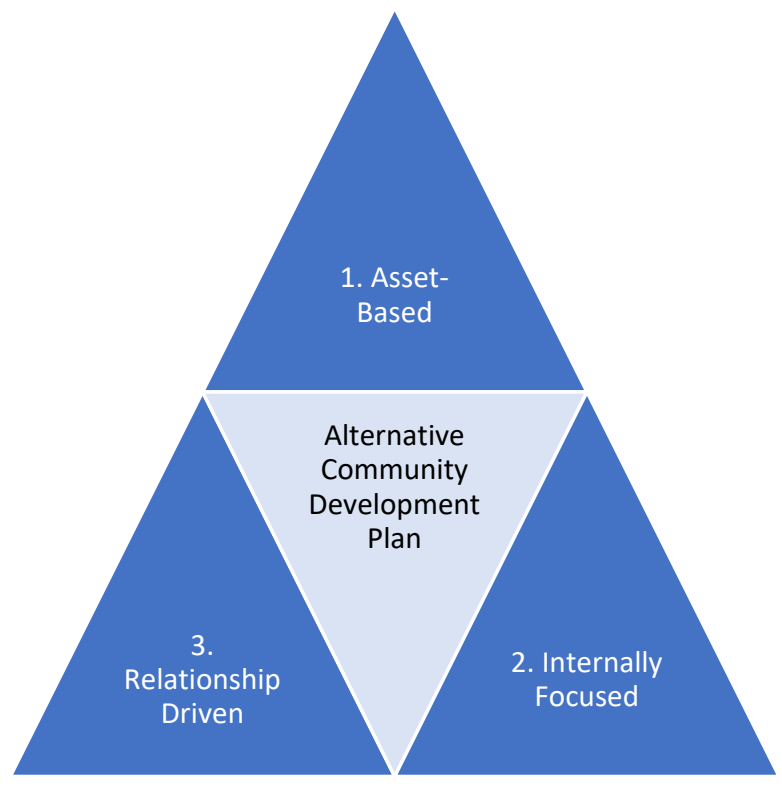

Figure 3. Three basic principles of an alternative community development plan. Source: Authors. 
Although $\mathrm{ABCD}$ has offered community development a whole host of tools for discovering and mobilizing assets, one of the criticisms of ABCD is that it has not said much about social injustice and systemic racism, sexism, and other forms of isms. More recently, the ABCD Institute released an organizational statement after George Floyd's killing in the hands of police in Minneapolis in 25 May 2020, acknowledging that, "Black and Brown people and Black and Brown communities are and have been under the occupation of systemic racism throughout American history for more than 400 years. We believe any community development work that does not acknowledge and address this reality is short sighted and will ultimately fail to build an authentic, sustainable, healthy community" [2].

The ABCD Institute faculty has recently written a number of publications on the topic of race, ethnicity, and social justice. For example, Jim Diers wrote a piece titled "Social justice is not as easy as $A B C D$ ", where he argues that "there is nothing inherently progressive about $\mathrm{ABCD}$. In fact, unless an $\mathrm{ABCD}$ approach is accompanied by a strong commitment to social justice and an understanding of what that entails, it has the potential to exacerbate current inequities" [3]. Indigo Bishop and Ron Dwyer-Voss in "Let's Get Explicit: Social Justice in Asset-Based Community Development" present the following argument:

Social justice frameworks and asset-based approaches to community development and community organizing overlap considerably. In fact, there is so much overlap that many longtime $A B C D$ practitioners presumed that $A B C D$ practice was a result of the civil rights movement and social justice work nationwide. Many thought "it goes without saying" that $\mathrm{ABCD}$ was rooted in social justice principle and anti-racism practices. But it doesn't go without saying. It must be said. We in the ABCD movement specifically, and the community development field in general, must be explicit if we are to avoid being complicit [4].

Finally, Dr. Ivis García et al. wrote "Planning With Diverse Communities", which discusses how $\mathrm{ABCD}$ can serve as a method for engaging diverse communities, particularly in urban planning practice [5]. Although all these writings are a good start to address head-on social justice issues, this Special Issue was born from the want of producing more publications on this topic. Below, we discuss in more detail the focus of this Special Issue "Enhancing the Role of Government, Non-Profits, Universities, and Resident Associations as Valuable Community Resources to Advance Equity, Access, Diversity, and Inclusion."

\section{On Diversity and $A B C D$}

As communities become increasingly diverse, practitioners, particularly in the public sector, are faced with not only rectifying the effects of past social injustice and mitigating systemic racism and sexism, but also addressing the needs of its most marginalized citizens, particularly African Americans. Efforts to mitigate past social injustices must also aim to promote equity in decision-making processes, where those groups that traditionally are excluded have access and agency to plan and envision a more equitable and just future. Moreover, to achieve these aims, more representation and agency of marginalized groups is central to this; however, it is an elephant in the room that the planning academia and practice have struggled to recruit and retain diversity and promote cultural competency. Across the nation, there are more and more institutional debates regarding how best to support diversity, equity, and inclusion within workplaces, institutions, organizations, and within our student and faculty bodies. However, there has been limited research that examines how practitioners, students, and faculty perceive or experience diversity within the urban planning field and the communities in which planners serve.

While previous research and case studies have sought to understand how government, non-profits, and universities can advance equity, more needs to be understood about how to better include, engage, and represent populations that historically have been left out of the decision-making process. This Special Issue will focus on challenges faced by local actors and innovative methods/solutions explored that support how to better engage and represent residents, neighborhood associations, and individuals in marginalized communities. Of particular interest are topics addressing the problems of segregation, gentrification, 
housing affordability, transportation access, education inequality, environmental justice, and political representation.

By improving the understanding of practitioners of the differences that exist between the variegated populations (e.g., older adults, young people, low-income individuals, immigrants, racial and ethnic minorities, LGBTQIA+ individuals, people with disabilities, to mention a few), the articles in this Special Issue outlined below have a tremendous amount of potential to examine and address issues of racism, marginalization, and discrimination.

\section{Outline of Article Contributions}

Institutional assets can make a difference in the lives of the most vulnerable. In "Welcome to Canada: Why Are Family Emergency Shelters 'Home' for Recent Newcomers?", authors Katrina Milaney, Rosaele Tremblay, Sean Bristowe, and Kaylee Ramage at the University of Calgary argue that the assets of institutions should better service homeless families, many of whom are also immigrant families. The authors suggest that although Canada is recognized internationally as a leader in immigration policy, institutions are not responsive to the traumatic experiences of many newcomers. Many mothers and children arriving in Canada are at elevated risk of homelessness. This study utilized a community-engaged design, grounded in a critical analysis of gender and immigration status. The authors conducted individual and group interviews with a purposive sample of 18 newcomer mothers with current or recent experiences with homelessness and with 16 service providers working in multiple sectors. Three main themes emerged from their study: (1) gendered and racialized pathways into homelessness; (2) system failures; and (3) pre- and post-migration trauma. This study revealed structural barriers rooted in preoccupation with economic success that negate and exacerbate the effects of violence and homelessness. In the conclusion, the authors note that the impacts of structural discrimination and violence are embedded in federal policy and that it is critical to posit gender and culturally appropriate alternatives that focus on system issues.

Dr. April Jackson (Florida State University), in "Three Local Organizing Strategies to Implement Place-Based School Integration Initiatives in a Mixed-Income Community," explores two policy efforts to revitalize public housing communities: education reform and HOPE VI. Chicago underwent a transformation of housing and schools from 2000 to 2014. Dr. Jackson examines the school integration planning efforts of three local actors in a Chicago neighborhood and asks how do actors make integration strategies work? This research investigates how efforts to remedy existing segregation in a Chicago neighborhood combined housing and school integration efforts through a single case study approach comprised of 20 in-depth interviews. Findings show that two approaches encouraged fairness in the residential mix, but did not promote an integrated educational experience. The third approach, a more ABCD approach, shows how a purposeful integration strategy works as part of a place-based effort. This study provides a lens to understand ongoing local community organizing efforts supporting education reform in a Chicago neighborhood and offers lessons learned by local actors about effective approaches to address the barriers to building mixed income communities.

Thinking about the fifth asset of economy and exchange, "An Asset-Based Perspective of the Economic Contributions of Latinx Communities: An Illinois Case Study," written by Dr. Ivis García (University of Utah) and Dr. Zafer Sönmez (The Conference Board of Canada), provides argumentation to policymakers who often need evidence that Latinx groups are an asset to their state economically. This article provides a case study in Illinois of how Latinx advocacy groups can help decision makers measure the Latinx share of economic activities and highlight its increasing role in the economic future of their state. The literature review provides a background on the growth of Latinx in the nation and state as well as how an asset-based approach is useful to estimate the contributions of Latinx. As a methodology, the authors use IMPLAN input-output models to calculate the economic footprint of Latinx in Illinois. In the findings, the authors first show the Latinx share of employment and sectoral distribution. Second, the authors demonstrate how this 
labor force has allowed the state to expand its production and purchasing power. In the conclusion, the authors discuss how this line of investigation allows us to explore what decision makers can do to facilitate a Latinx action agenda from the asset-based perspective.

In regards to the sixth $\mathrm{ABCD}$ community asset, culture, history, and stories, "Storytelling and Arts to Facilitate Community Capacity Building for Urban Planning and Social Work," by Dr. Crystal Taylor (Florida State University) and Dr. Qinghong Wei (Story Bridge), takes readers to a community land trust in Lopez Island, Washington. Facing sustainable housing and agricultural issues, the land trust brought in a two-day arts and storytelling program to build support for their ecosocial community work. In order to help community groups apply for art program funding, the authors piloted a survey to evaluate the experience of workshop participants by folding in community capacity measures from the public health literature. Preliminary results show that the storytelling program makes strides in deepening connections to others and generating authentic dialogue. Participants reported both positive experiences of building trust and negative feelings of vulnerability. Though the facilitators brought unique theatrical and choreographic skills to the programming, planners and social workers can take away for practice a simple storytelling exercise that participants enthusiastically expressed fostered listening, trust, and connection. The authors hope that this article encourages readers of Societies to consider supporting arts and storytelling programming as an innovative approach to building capacity for community-driven work.

Thinking collectively about the ABCD approach, Dr. Zechariah Lange (Florida State University), in "Bridges Don't Make Themselves: Using Community-Based Theater (CBT) to Reshape Relationships-Rethinking the Idea of Abundance in ABCD," builds on the core tenets of $A B C D$ to enhance the framework of economic scarcity towards co-created relational 'abundance' at the neighborhood scale. This work comprises a multiple case study of two community-based theaters in Middle Appalachia and the Gulf Coast of Florida. Based on 25 in-depth interviews and relational participatory action research, Lange spent six months assisting, building, and acting in performances to document the creation and enactment of performances. Building on this perspective, Dr. Lange shows how the two theaters simultaneously catalyze relationship and alter how relationships are experiences to engage community members in discussion and performances that manifest dimensions of 'abundance', which also expand upon the normative conceptions of asset-based community development. Moreover, the work highlights how CBTs are functioning places of ABCD within a more realized and described context.

Dr. Andrea Roberts (Texas A \& M), in "Preservation without Representation: Making CLG Programs Vehicles for Inclusive Leadership, Historic Preservation, and Engagement," examines public historic preservation agencies' ability to support social inclusion aims within the context of the Certified Local Government (CLG) program in Gonzales, Texas. CLG programs are federally funded and provide technical assistance, grants, and loans to communities. Through surveys and questionnaires collected on self-assessment activities during pilot training on implicit bias, outreach, and cultural resource surveying, Dr. Roberts evaluated the survey results in order to understand the challenges around ways to diversify organizational leadership. After comparing the results of these two government assessment tools, she found that more creatively designed training and capacity building is necessary around inclusion, identifying structural barriers to participation, foundational knowledge of historic preservation and planning practice, and ethics.

In "Gown Goes to Town: Negotiating Mutually Beneficial Relationships between College Students, City Planners, and a Historically Marginalized African-American Neighborhood," authors Dr. April Jackson, Dr. Tisha Holmes, and Dr. Tyler McCreary at Florida State University examine a university-community partnership between Florida State University (FSU) and the Griffin Heights neighborhood. This article traces the 9month collaboration between the City of Tallahassee, the three community-engaged courses in the FSU Department of Urban Planning, the Griffin Heights Neighborhood Association, and various community stakeholders to develop a neighborhood plan for the predomi- 
nantly African American community of Griffin Heights. The authors found that although city planners have increasingly adopted the language of community engagement, many planning processes remain inflexible, bureaucratic, and under resourced. To reconcile these limitations, the authors argue for mutually beneficial collaboration that provides a more equitable and just approach to community engagement that is adaptable, culturally competent, and has clear commitments to long-term funding and project implementation. This work is a stark reminder that while community-engaged courses create opportunities to facilitate community empowerment, they also at times risk perpetuating the disenfranchisement of African American community members in city planning processes.

Churches are central to asset-based community work. In "Pushing Back on Displacement: Community-Based Redevelopment through Historically Black Churches," authors Brandon Born, Olivia Baker, Mark Jones, Donald King, and Dylan Marcus investigate the challenges associated with neighborhood revitalization that can oftentimes result in gentrification and subsequent displacement of existing low-income residents, particularly communities of color. In this paper, the authors offer a community-based approach to redevelopment through a collaboration between two courses at the University of Washington, the Nehemiah Initiative, the City of Seattle, and various community partners in real estate and housing. This partnership situates the historic Black churches as a way to counter impending displacement and cultural removal in Black communities. The authors trace the historically Black neighborhood, the Central District in Seattle, and the formation of the Nehemiah Initiative to understand the collaborative effort and potential ways to mitigate displacement pressures and the loss of cultural resources, spaces, and community. Overall, this work provides opportunities and recommendations for practice and policy towards more community-responsive redevelopment practices from both the public sector and non-profit organizations.

How can anchor institutions such as universities use their assets to help residents to stay in place as opposed to contribute to their displacement? In "Student Housing Choices and Neighborhood Change: Brown University 1937-1987," authors Nathaniel Philip Petit and Marijoan Bull examine the case study of Brown University in Providence, Rhode Island, to understand how anchor institutions exacerbate residential displacement pressures. The authors present a case study that documents the history of Brown University's institutional decision-making through enrollment expansion, real estate decisions, and student residency policies that supported student housing redevelopment in a working-class community of color that displaced existing residents. Utilizing primary source archival data, the authors tell the story of neighborhood transformation in the context of resident concerns and pushback around increasing displacement pressures driven by Brown University over a 50-year period. This case offers lessons on ways to more effectively center equity in anchor institution works by integrating community stakeholders in decision-making processes to balance competing interests and confronting direct and indirect sources of power and influence.

Finally, authors Claire Jane Snowdon and Leena Eklund Karlsson (University of Southern Denmark, Odense, Denmark), in "A Critical Discourse Analysis of Representations of Travelers in Public Policies in Ireland," discuss how in Ireland, negative stereotypes of the Traveler population have long been a part of society. The beliefs that surround this minority group may not be based in fact, yet negative views persist such that Travelers find themselves excluded from mainstream society. The language used in discourse plays a critical role in the way Travelers are represented. Their study analyses the discourse in the public policy regarding Travelers in the National Traveler and Roma Inclusion Strategy (NTRIS) 2017-2021. This study performs a critical discourse analysis (CDA) of the policy with the overall aims of showing signs of the power imbalance through the use of language and revealing the discourses used by elite actors to retain power and sustain existing social relations. The key findings show that Travelers are represented as a homogenous group that exists outside of society. They have no control over how their social identity is constructed. The results show that the constructions of negative stereotypes are inter- 
textually linked to previous policies, and the current policy portrays them in the role of passive patients, not powerful actors. The discursive practice creates polarity between the "settled" population and the "Travelers," who are implicitly blamed by the state for their disadvantages. Through the policy, the government disseminates expert knowledge, which legitimizes the inequality and supports this objective "truth." The authors argue that this dominant discourse, which manifests in wider social practice, can facilitate racism and social exclusion. Their study highlights the need for Irish society to change the narrative to support an equitable representation of Travelers.

\section{Conclusions}

In addition to understanding underlying issues that reproduce systemic injustice and inequality in low-income but otherwise resourceful communities, this Special Issue has sought to discover and mobilize community assets as well as provide innovative tools for those seeking to engage with the growing racial diversity of cities. We hope that the ABCD stories, practices, and techniques presented in this Special Issue can be used to identify patterns of racism, ethnocentrism, classism, and other patterns of oppression or bias within our culture and to find common language to discuss and strengthen a shared culture and commitment to actively deconstructing them-both so that we can stop perpetuating them and can stop excluding/oppressing those currently working in various positions in the city, but also so that we can more effectively grow an inclusive city where anyone joining can contribute, lead, and expand their practice fully.

Author Contributions: I.G. wrote the original draft and A.J. reviewed and edited this article. All authors have read and agreed to the published version of the manuscript.

Funding: This research received no external funding.

Acknowledgments: In this section we acknowledge MDPI editors Alice Li and Vivian Zhang for their dedication and kind assistance making this Special Issue possible.

Conflicts of Interest: The authors declare no conflict of interest.

\section{References}

1. Kretzmann, J.P.; McKnight, J. Building Communities from the Inside out: A Path toward Finding and Mobilizing a Community's Assets; The Asset-Based Community Development Institute, Institute for Policy Research, Northwestern University: Evanston, IL, USA, 1993.

2. ABCDI. Asset Based Community Development Institute Policy Statement on Anti-Racism. 2020. Available online: https: / / resources.depaul.edu/abcd-institute/Pages/default.aspx (accessed on 1 April 2021).

3. Diers, J. Social Justice Is Not as Easy as ABCD. Neighbor. Power Blog. 2020. Available online: http://blog.neighborpower.org/ uncategorized/social-justice-is-not-as-easy-as-abcd/\#: \{\}:text=In\%20fact\%2C\%20unless\%20an\%20ABCD,potential\%20to \%20 exacerbate\%20current\%20inequities (accessed on 1 April 2021).

4. Bishop, I.; Dwyer-Voss, R. Let's Get Explicit: Social Justice in Asset-Based Community Development. Shelterforce: The Voice of Community Development. 2019. Available online: https://shelterforce.org/2019/04/26/lets-get-explicit-social-justice-in-assetbased-community-development/ (accessed on 1 April 2021).

5. García, I.; Garfinkel-Castro, A.; Pfeiffer, D. Planning with Diverse Communities; Planning Advisory Service (PAS) Report 593; American Planning Association: Chicago, IL, USA, 2019. Available online: https://www.planning.org/publications/report/9165 143/ (accessed on 1 April 2021). 